\title{
LAS PYMES Y LA POLÍTICA PÚBLICA AMBIENTAL EN COLOMBIA: REDIRECCIONAMIENTO CON BASE EN LA CULTURA ORGANIZACIONAL ${ }^{1}$
}

\author{
Por: Pedro A. Bohórquez Pulido ${ }^{2}$ y Juan P. Cendales Rodríguez ${ }^{3}$
}

\begin{abstract}
RESUMEN
En el artículo se propone evaluar los cimientos de la política pública ambiental, para determinar los factores que podrían promover un re-direccionamiento de la misma al interior de las pequeñas y medianas empresas PYMEs, en congruencia con el tipo de cultura que se observa en ellas. Para ello, se llevó a cabo una revisión bibliográfica de las propuestas sobre cultura organizacional, las características que la definen y su rol en el direccionamiento estratégico corporativo; de esa forma se procedió a evaluar la política pública ambiental de Colombia en contraste con recomendaciones de organismos multinacionales (como la OCDE), de tal forma que se pudieran identificar los problemas relacionados con su origen y aplicabilidad. Como conclusión, la política pública ambiental carece de un sistema de incentivos adecuado para propiciar el desarrollo de comportamientos amigables con el ambiente y, en ese sentido, desconoce el engranaje cultural de las PYMEs que se orienta al logro de objetivos financieros como el de supervivencia y crecimiento
\end{abstract}

Palabras clave: PYME, Gestión Ambiental, Cultura Organizacional, Política Pública

JEL: Q58

1. Este documento es producto del proyecto de investigación "INCLUSIÓN DE LINEAMIENTOS AMBIENTALES EN LA CULTURA ORGANIZACIONAL PARA UNA GESTIÓN AMBIENTAL EMPRESARIAL COMPROMETIDA CON LA SUSTENTABILIDAD” (Código HERMES Universidad Nacional de Colombia 21619 - 23616), como parte del programa jóvenes investigadores de COLCIENCIAS - 2013. Los investigadores hacen parte del Grupo de Estudios Interdisciplinarios en Gestión y Contabilidad - INTERGES.

2. Contador Público y candidato a Magíster en Ciencias Económicas de la Universidad Nacional de Colombia. Correo de contacto: peabohorquezpu@unal.edu.co

3. Contador Público y candidato a Magíster en Administración de la Universidad Nacional de Colombia. Correo de contacto: jpcendalesr@unal.edu.co

Artículo recibido: 9 de febrero de 2015. 


\title{
SMES AND ENVIRONMENTAL PUBLIC POLICY IN COLOMBIA: REDIRECTION BASED ON ORGANIZATIONAL CULTURE
}

\author{
By: Pedro A. Bohórquez Pulido y Juan P. Cendales Rodríguez
}

\begin{abstract}
This document aims to evaluate the foundations of environmental public policy, to determine the factors that could promote the redirection inside SMEs, according to their organizational culture. The authors made a literature review about organizational culture proposals, characteristics, definitions, and its role in strategic organizational direction; then we evaluated the environmental public policy in Colombia in contrast to multinational organizations recommendations (such as OECD), and finally we identified problems related to the causes and applicability of policies. As a conclusion, environmental public in policy in Colombia lacks a proper incentive system to promote environmental friendly behaviors; in that sense, organizational culture in SMEs, which follows financial goals, is ignored.
\end{abstract}

Key words: SME, Environmental Management, Organizational Culture, Public Policy

JEL: Q58 


\title{
PME E PÚBLICO AMBIENTAL POLÍTICA NA COLÔMBIA: REDIRECTION COM BASE NA CULTURA ORGANIZACIONAL
}

\author{
Por: Pedro A. Bohórquez Pulido y Juan P. Cendales Rodríguez
}

\section{RESUMO}

Este documento tem como propósito avaliar os cimentos da política pública ambiental, para determinar os fatores que poderiam promover uma re-direcionamento da mesma ao interior das pequenas e medianas empresas (PYME), em congruência como o tipo de cultura que é observada nelas. Para conseguir este objetivo, se realizou uma revisão bibliográfica das propostas sob a cultura organizacional, as características que as definem e seu rol no direcionamento estratégico corporativo; desta forma, foi avaliada a política pública ambiental da Colômbia em contraste com recomendações de organizações multilaterais (como a OCDE), de tal forma que for possível uma identificação dos problemas relacionados à origem e aplicabilidade. Como conclusão, a política pública ambiental carece de um sistema de incentivos convenientes para propiciar o desenvolvimento de comportamentos amigáveis com o ambiente e nesse sentido desconhece a engrenagem cultural das PYMES que está orientado ao logro de objetivos financeiros, supervivência e desenvolvimento.

Palavras chaves: PYME, gestão ambiental, cultura organizacional, política pública.

JEL: Q58. 


\section{INTRODUCCIÓN}

La gestión ambiental en las organizaciones es, hoy por hoy, un tema que ha suscitado gran debate entre empresarios, académicos y grupos sociales que han empezado a notar la importancia de que las empresas asuman la responsabilidad del impacto ambiental que producen. En ese sentido, el llamado para las compañías ha sido enfocado en la adopción de comportamientos más amigables con el medio ambiente, como parte de su quehacer productivo.

Si bien es cierto que los grados de concientización frente a lo medioambiental han aumentado en los últimos años, especialmente como consecuencia de fenómenos naturales que han demostrado los nocivos efectos de la intervención humana en el ambiente, aún hay mucho por resolver para transitar hacia lo que se ha denominado el desarrollo sostenible, término que resulta muy usual en los instrumentos de política pública de diversos países.

La política pública ambiental ha estado caracterizada por una ausencia de integralidad y una aplicabilidad limitada, que se deriva de la quebrantada institucionalidad de las autoridades ambientales y un deficiente entendimiento de los incentivos que pueden conducir a la adopción de lineamientos ambientales. Mientras el Gobierno se preocupe por emitir normatividad sin planear los escenarios de su aplicación, lo único que se obtendrá es un robusto aparato normativo que dista de ser un adecuado instrumento de gobernabilidad y control.

El problema de la proposición de políticas públicas se acentúa cuando se desconoce el tipo de cultura que predomina en las empresas, particularmente en las pequeñas y medianas - PYMEs, donde los objetivos de índole financiero son los que promueven el quehacer organizacional. Aunque el objetivo más ambicioso, en términos ambientales, sería crear la conciencia suficiente sobre los impactos de las organizaciones en la naturaleza, los esfuerzos deberían concentrarse en, al menos, lograr que las empresas tengan una mayor inclinación por la preservación de los recursos naturales.

Por lo anterior, resulta imprescindible que se reconozca y evalúe el tipo de cultura organizacional, de tal forma que se puedan escoger los mecanismos idóneos para la adopción de comportamientos amigables con el ambiente y sistemas de gestión ambiental. En ese sentido, el propósito que se persigue con este trabajo es el de proponer un re-direccionamiento a las políticas públicas ambientales, de tal forma que se genere un sistema de incentivos apropiado que, de la mano con la cultura organizacional predominante en las PYMEs, conduzca al mejoramiento del desempeño ambiental organizacional.

\section{MARCO TEÓRICO Y ESTADO DEL ARTE}

\subsection{CULTURA ORGANIZACIONAL}

Existen diversas definiciones para el término Cultura Organizacional (en adelante CO), las cuales surgen a raíz de diversas fuentes de información y enfoques. A través de la historia se han destacado algunos autores como Smircich (1983), Schein (2004), Linnenluecke \& Griffiths (2010), y Frassa (2011), quienes han argumentado 
como la CO puede ser definida desde diferentes frentes a través de la compilación y clasificación de teorías al respecto, mientras otros teóricos se han preocupado en dar definiciones con base en diferentes enfoques, tales como Hofstede (1990), Trice \& Bayer (1993), Schultz (1995), Deal \& Kennedy (1999) (citados en Schein, 2004: 12), Cameron y Queen (1999) y otros.

Una de las definiciones de cultura organizacional que más divulgación ha tenido, es la propuesta por Schein (2004), que no sólo incluye los ambientes interno y externo de una organización, sino que también condiciona los elementos de la cultura a la posibilidad de que estos conduzcan al éxito corporativo:

La cultura de un grupo puede ser definida como el patrón de supuestos básicos compartidos que son aprendidos por una colectividad para resolver sus problemas de adaptación externa e integración interna, que han funcionado muy bien para ser considerados como válidos y, por lo tanto, ser enseñados a los nuevos miembros como una forma correcta de percibir, pensar y sentir en relación a dichos problemas (Schein, 2004: 17).

La importancia de la definición anterior radica en la forma como se vincula la construcción colectiva de la cultura a la utilidad que ésta tenga, para la resolución de conflictos dentro de la organización. Además se define por el éxito que promueva en el desempeño organizacional. Esta definición de CO será citada y ampliada posteriormente y será utilizada como base del presente documento.

Con base en el autor, la cultura se configura a través de niveles, los cuales permiten distinguir rasgos distintivos que caracterizan la cultura organizacional tanto en cada organización, como al interior de las mismas, estos son: artefactos, creencias y valores compartidos, y supuestos básicos (Schein, 2004: 25-30). Simultáneamente, se puede dar el caso de que coexistan en la organización diferentes culturas que interactúen entre sí; dándose 3 niveles de interacción: integración, diferenciación, y la fragmentación (Ibíd.: 200). Unido a esto, Howard \& Grenville (2006:66) proponen una teoría de lentes, en la que se muestra como las diversas posturas culturales pueden converger o divergir ante la toma de decisiones.

La cultura organizacional puede tener ciertos rasgos y patrones que permiten ubicarla dentro de grupos culturales más amplios. Ésta es una forma de definir el Marco de Valores en Competencia planteado inicialmente por Cameron \& Quinn (1999), el cual tipifica las clases de cultura que se pueden encontrar al interior de las organizaciones, de acuerdo con dos dimensiones básicas:

- La primera evalúa si la organización está enfocada hacia la flexibilidad y el dinamismo en sus procesos internos, o si es partidaria de los esquemas de control exhaustivo y estabilidad.

- La segunda considera si la organización tiene en cuenta su medio ambiente externo o si sólo está interesada en el desempeño de su medio ambiente interno.

Derivado del modelo inicial de Cameron \& Quinn, integran posturas conceptuales, haciendo una descripción más integradora de las distintas percepciones 
acerca de las culturas imperantes en una organización (Linnenluecke \& Griffiths, 2010:359-360). Dicho modelo esquematiza los tipos de cultura en 4 áreas (ver gráfico 1). Para el caso de este documento se tomará la interpretación dada por Linnenluecke \& Griffiths (2010) con base en la propuesta de Jones et al. (2005) y Zammuto et al. (2000):

\section{Gráfico 1: Tipos de cultura organizacional}

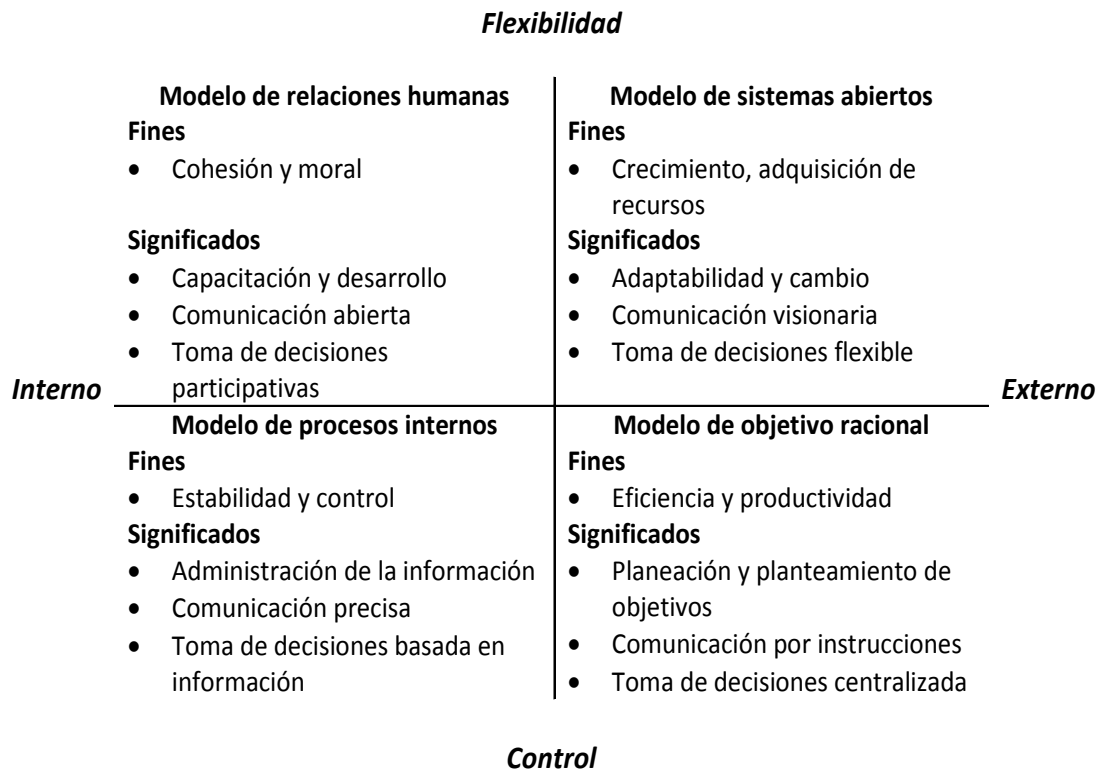

Fuente: Traducido de Linnenluecke \& Griffiths, 2010, p. 359; Modificado de Jones et al. (2005) \& Zamnuto et al. (2000); citado en: Linnenluecke \& Griffiths, 2010, p. 359.

Estas consideraciones sobre los tipos de cultura que pueden estar presentes en las organizaciones, se deben ahora contextualizar para el caso particular de las pequeñas y medianas empresas PYMEs, ya que alrededor de ellas se encuentra el objetivo de este trabajo.

\subsection{CULTURA ORGANIZACIONAL EN PYMES}

Desde el año 2005 se ha incrementado la preocupación por parte de los académicos, sobre el desempeño ambiental de las PYMEs, lo que coincide con la entrada en vigor del Protocolo de Kyoto y la difusión por parte de GRI (Global Reporting Initiative) de las guías para la evaluación del desempeño económico, social y ambiental de las PYMEs (Herrera, Larrán y Martínez-Martínez, 2012: 42).

Se han adelantado estudios que pretenden vincular los atributos y características de los empresarios, sus niveles éticos y las capacidades que tienen para influir en las decisiones, con el comportamiento de la empresa y su papel en la sociedad 
(Guth \& Tagiuri, 1965; Carroll y Hoy, 1984; Barnett y Karson, 1987; De Jong et al., 2008; Nielsen \& Thomsen, 2009; Godos-Díez et al., 2011 citados en Herrera, Larrán y Martínez-Martínez, 2012).

Lo anterior resulta trascendental para entender la dinámica de las PYMEs, si se tiene en cuenta que, cuando se constituye una, quien asume el liderazgo y propone las declaraciones formales de misión y visión, las políticas, los valores, las directrices y lineamientos de acción, es precisamente el propietario gestor, quien es el que invierte capital y gestiona las diversas operaciones.

En palabras de Shein (2004): el fundador individual -ya sea un empresario o el que convoca a un nuevo grupo- tendrá una serie de visiones personales, metas, creencias, valores y concepciones sobre cómo deberían ser las cosas (p. 15). En ese sentido, el fundador de una PYME transmite sus ideas sobre la forma cómo se podría llegar al éxito, la manera de alcanzar los objetivos organizacionales, los métodos para sobrevivir en el mercado, etc.

En los niveles de análisis cultural propuestos por Schein (2004) (y mencionado anteriormente por los autores), resultaba claro que los valores y creencias compartidos son manifestaciones de lo que se considera correcto o apropiado frente a aquello que no lo es. Cuando estas creencias se materializaban en acciones que conducían a una situación de éxito, era probable que la colectividad legitimara dichas creencias y poco a poco transitaran hacia un estado de supuestos básicos.

Estableciendo una analogía, el propietario gestor de las PYMEs es el llamado a proponer estos cursos de acción e insertarlos en la estrategia de la empresa, de tal forma que las situaciones de éxito se deriven de sus decisiones. En pocas palabras, el propietario gestor impregna su visión de negocio en la estrategia corporativa.

Las diversas visiones de los socios configuran la cultura organizacional en las PYMEs, la cual puede hacerse evidente de manera formal o informal Wallace et al (1999). La cultura formal se entiende como las declaraciones idealizadas acerca de las creencias y comportamientos que deberían existir, usualmente manifestadas a través de la misión y la visión, las políticas, los procedimientos y las reglas (Balzarova, Castka, Bamber y Sharp, 2006: 92); y la cultura informal se define como "los comportamientos y creencias actuales" (Ibíd.: 92)

De esta forma se crea una cultura en común que se promueve desde el socio gestor y se extiende a los demás integrantes de la empresa (Rodríguez, 2009, p. 73). El desarrollo de valores y creencias compartidos, de acuerdo con los postulados de Schein, se concreta con la definición de la estrategia corporativa que obedece a las concepciones del socio gestor. En resumidas palabras, es el socio gestor el que imprime en la cultura los parámetros que considera necesarios para que la compañía crezca y se consolide.

Si bien este proceso de construcción cultural puede significar la creación de conflictos entre la cultura de los dirigentes y la de los dirigidos, la tendencia es a que se implementen los parámetros culturales del socio gestor, teniendo en cuenta 
los atributos de poder que le son inherentes (Aktouf, 2002, p. 30). La convergencia termina siendo un resultado de esta dinámica (Jenkins, 2009, p. 244).

Como parte de la planificación estratégica adelantada por los socios gestores, se presenta la construcción de un engranaje cultural que obedece a los objetivos de supervivencia empresarial y crecimiento en el mercado. Cada socio gestor procura potenciar los resultados de su inversión y, en ese sentido, toma decisiones que conduzcan a un mejor desempeño organizacional. Ante este comportamiento, es natural que toda iniciativa ambiental o de responsabilidad social que sea adoptada, deba contribuir a la consolidación de las metas económicas (Herrera, Larrán\& Martínez-Martínez, 2012).

Los autores llevaron a cabo un análisis de estudios de caso colombianos y del exterior (Ver Tabla 1), como los adelantados por Bianchi \& Noci (1998), Uhlaner et al. (2011), Schlierer et al. (2012), Gálvez y García (2011) y Herrera, Larrán y Martínez-Martínez (2012), con los cuales resultaba evidente el vínculo entre iniciativas medioambientales y mejoramiento del desempeño económico.

Tabla 1: Tipos de cultura organizacional

\begin{tabular}{|c|c|c|}
\hline Autor & Objeto de estudio & Conclusión \\
\hline $\begin{array}{l}\text { Bianchi \& Noci } \\
\text { (1998) }\end{array}$ & 46 PYMEs italianas & $\begin{array}{l}\text { Labajapresióndelosstakeholders, lacomplejidad } \\
\text { en la implementación de dichas estrategias y la } \\
\text { alta demanda de recursos (humanos, físicos, } \\
\text { económicos) son los principales motivos para } \\
\text { no cambiar sus estrategias (p.272). Los autores } \\
\text { partieron de que los propietarios de las PYMEs } \\
\text { tienden a tomar sus decisiones empresariales } \\
\text { con base en objetivos de desempeño financiero, } \\
\text { ya que persiguen propósitos de sostenimiento y } \\
\text { crecimiento organizacional. Las PYMEs buscan } \\
\text { algún grado de beneficio económico (bien sea en } \\
\text { términos de producción o de ventas), prefieren } \\
\text { inversiones de corto plazo que representen } \\
\text { altos retornos y concentran sus esfuerzos en la } \\
\text { supervivencia del día a día (KPMG, 1997; citado } \\
\text { en Bianchi \& Noci, 1998) }\end{array}$ \\
\hline $\begin{array}{l}\text { Uhlaner, } \\
\text { Berent-Braun, } \\
\text { Jeurissen \& Wit } \\
\text { (2012) }\end{array}$ & 689 PYMEs alemanas & $\begin{array}{l}\text { Entre mayores son los beneficios financieros } \\
\text { recibidos por conservar recursos naturales y } \\
\text { energéticos, mayor es la probabilidad de que } \\
\text { las PYMEs se relacionen con prácticas de gestión } \\
\text { ambiental. Sin embargo, aspectos del sector } \\
\text { en el que se desempeña la PYME, también } \\
\text { influencian fuertemente la implementación de } \\
\text { prácticas ambientales (pp. 422-423). }\end{array}$ \\
\hline
\end{tabular}




\begin{tabular}{|c|c|c|}
\hline $\begin{array}{l}\text { Schlierer, } \\
\text { Werner, Signori, } \\
\text { Garriga, } \\
\text { Weltzien, Van } \\
\text { Rossem \& } \\
\text { Fassin (2012) }\end{array}$ & $\begin{array}{l}123 \text { socios gestores de } \\
\text { PYMEs europeas }\end{array}$ & $\begin{array}{l}\text { Este estudio analizó la percepción de los socios } \\
\text { hacia los stakeholders que en su mayoría } \\
\text { se limitaban a los proveedores, clientes y } \\
\text { empleados; lo que se puede interpretar como } \\
\text { que las conclusiones halladas aplican solo } \\
\text { para una gestión responsable al interior de la } \\
\text { empresa y las cadenas de valor. En países como } \\
\text { Italia, Francia o Noruega las empresas velaban } \\
\text { por los intereses de los stakeholders, mientras } \\
\text { que en lugares como Reino Unido o España } \\
\text { las decisiones eran dirigidas principalmente al } \\
\text { mercado y los inversionistas. (p. } 40-46) \text {. }\end{array}$ \\
\hline $\begin{array}{l}\text { Gálvez y García } \\
\text { (2011) }\end{array}$ & $\begin{array}{l}60 \text { MIPYMEs de } \\
\text { mediana o alta } \\
\text { tecnología en Cali- } \\
\text { Colombia }\end{array}$ & $\begin{array}{l}\text { Los resultados obtenidos mostraron un } \\
\text { vínculo significativo entre la cultura jerárquica } \\
\text { (propuesta por Cameron \& Quinn) y el modelo } \\
\text { de procesos internos (propuesto por Jones et al., } \\
2005 \text { \& Zammuto et al., 2000), que denotan una } \\
\text { cultura orientada a los formalismos, el control, la } \\
\text { impersonalidad, entre otros. }\end{array}$ \\
\hline $\begin{array}{l}\text { Herrera, Larrán } \\
\text { y Martínez- } \\
\text { Martínez (2012) }\end{array}$ & $\begin{array}{l}\text { Revisión bibliográfica } \\
\text { de la relación entre } \\
\text { responsabilidad social } \\
\text { y performance en las } \\
\text { pequeñas y medianas } \\
\text { empresas }\end{array}$ & $\begin{array}{l}\text { Del documento se aprecian conclusiones y } \\
\text { estudios citados (especialmente Fitzgerald et } \\
\text { al. (2010), Niehm et al. (2008) y Hamma et al. } \\
\text { (2009)) que evidencian un interés financiero de } \\
\text { las PYMEs en la toma de decisiones dirigidas al } \\
\text { cuidado ambiental: "Es de apreciar que, aunque } \\
\text { existen distintas teorías y perspectivas a través } \\
\text { de las cuales los autores han argumentado que } \\
\text { la responsabilidad social empresarial contribuye } \\
\text { al desarrollo de ventajas competitivas y a } \\
\text { la creación de valor, todos comparten una } \\
\text { racionalidad económico-estratégica centrada en } \\
\text { la generación de ganancias a partir de prácticas } \\
\text { amigables con el ambiente" (Herrera, Larrán y } \\
\text { Martínez-Martínez, 2012) }\end{array}$ \\
\hline
\end{tabular}

Fuente: Elaboración propia.

En concordancia con el postulado de Jones et al. (2005) \& Zammuto et al. (2000), en referencia al modelo de procesos internos, en el cual se da prioridad a las metas de índole económico y se desconoce los efectos del medio ambiente externo, lo que los autores pudieron apreciar fue su sintonía con el tipo de cultura predominante en PYMEs y extendida por el socio gestor, es decir, es ese tipo de cultura el que haya correspondencia con el engranaje cultural que es transmitido desde el socio gestor.

En ese orden de ideas, los autores llegaron a la conclusión de que la cultura predominante en PYMEs corresponde a la cultura descrita en el modelo de procesos internos, en el que se da prioridad a la estabilidad y al control permanente. 
Por ello, cualquier iniciativa de gestión ambiental debe atender los objetivos de crecimiento económico. Si no hay los incentivos adecuados, tales iniciativas no serán consideradas como parte integral de la planeación estratégica.

\subsection{ACUERDOS AMBIENTALES: EL PROTOCOLO DE KYOTO}

El Protocolo de Kyoto es un acuerdo internacional que constituye el primer instrumento legal por el cual se establece el compromiso de limitar los gases de efecto invernadero en los países desarrollados y con economías de transición de mercado (Sagrera, 2011). Fue firmado en la ciudad de Nueva York en 1992, adoptado en 1997 durante la tercera cesión de la Conferencia de las Partes (COP34) de la Convención Marco de las Naciones Unidas sobre Cambio Climático y entró en vigor el 16 de febrero de 2005 (Sagrera, 2011).

En este acuerdo los países desarrollados y aquellos con economías en transición se comprometen para el periodo comprendido entre el año 2008 al 2015, a reducir o limitar las emisiones de gases de efecto invernadero (GEIs) en un 5,2\% en comparación con las emisiones base de 1990, con el objetivo de promover el desarrollo sostenible. Esta reducción debe lograrse en un plazo suficiente para permitir que los ecosistemas se adapten naturalmente al cambio climático, se asegure que la producción de alimentos no se vea amenazada y se haga posible un desarrollo económico sostenible (García \& Cuesta, 2007). Los gases de efecto invernadero incluidos son: dióxido de carbono (CO2), metano (CH4), óxido nitroso (N2O), hidrofluorocarbonos (HFCs), perfluorocarbonos (PFCs) y hexafluoruro de azufre (SF6). Cuando se exceda la "cantidad atribuida" o emisión permitida de estos gases, se incurrirá en incumplimiento del convenio (Sagrera, 2011).

Han ratificado o firmado el acuerdo150 países, tomando las obligaciones de Kyoto como una guía de su política ambiental. Estados Unidos y Australia no se han incorporado y han expresado su voluntad de impulsar políticas internas que respondan a sus obligaciones como firmantes de la Convención marco de las Naciones Unidas para el cambio climático a través de medidas unilaterales (Laferriere, 2008).

Son estructurados tres mecanismos de flexibilización a través de los cuales se disminuyen los costos de reducción de las emisiones de GEIs (Lamarca Junior \& Leite da Silva, 2009):

- Mecanismo de desarrollo limpio (MDL), para proyectos implementados conjuntamente entre países desarrollados y en vía de desarrollo.

- Implementación conjunta, donde se suministran Unidades de Reducciones Certificadas de Emisiones (RCE) a los países industrializados que realizan proyectos conjuntos para reducir sus emisiones de GEIs (García \& Cuesta, 2007).

- Certificados de transferencia y comercialización de reducción de emisiones entre países desarrollados.

Los anteriores mecanismos buscan explotar las oportunidades de reducir costos de mitigar las emisiones de GEIs permitiendo que esas reducciones sucedan en los países donde el costo marginal por tonelada de emisiones reducidas sea menor 
(Acquatela, 2001). Favorecen también el surgimiento del mercado del carbono, permitiendo que los países desarrollados disminuyan los costos requeridos para alcanzar las metas de reducción. Así mismo, crea la posibilidad de que los países con economías en transición reciban ganancias a través de proyectos alrededor del mercado del carbono con tecnologías más limpias (Lamarca Junior \& Leite da Silva, 2009).

Para el caso de Colombia, este protocolo fue ratificado mediante la Ley 629 del año 2000, donde el único mecanismo aplicable es el MDL, ya que facilita que los países desarrollados y grandes emisores de GEIs inviertan sus recursos en países en vía de desarrollo, a través de la compra de Certificados de Reducción de Emisiones (CRE) (Monroy \& Aguirre, 2003).

\subsection{POLÍTICAS PÚBLICAS AMBIENTALES EN COLOMBIA}

En Colombia se aprecia un problema relacionado con la contundencia y la integralidad de las políticas públicas, pues a pesar de que existen organismos de control capaces de generar un aparato normativo adecuado para los intereses del país, no hay la suficiente cohesión entre los entes encargados de supervisar la preservación de los recursos naturales.

A pesar de que el desarrollo sostenible es una prioridad manifiesta en la Constitución Política de Colombia (1991: Art. 339), la forma cómo se ha tratado de dar respuesta a la necesidad de un mejor cuidado del medio ambiente, no ha sido la más apropiada. Hoy por hoy, ha sido notable el requerimiento de incorporar un mayor número de lineamientos ambientales en distintos sectores (incluyendo al empresarial), y este esfuerzo se ha visto materializado en propuestas como la del Plan Nacional de Desarrollo (PND) 2010-2014, en el cual se procura vincular el desarrollo económico con el cuidado medioambiental (OECD/ECLAC, 2014, p. 52).

Si bien el PND considera, dentro de su articulado, la conveniencia de impulsar las locomotoras de crecimiento en conjunto con el cuidado del medio ambiente, no es clara la forma cómo los sectores productivos van a evaluar el mejoramiento de su desempeño ambiental. Lo anterior hace que, para tales sectores, no sea relevante incluir en la evaluación de su desempeño lo relacionado con el desarrollo sostenible. Así, entonces, se supedita lo ambiental a lo económico, una situación que ya se había advertido en el análisis de la cultura organizacional en PYMEs.

Desde el año 2003, han sido numerosos los decretos, leyes, resoluciones, entre otros que, al igual que el PND, han pretendido incluir en las empresas unos procedimientos concisos para mejorar el cuidado del ambiente. Lo que ha resultado más evidente aún, es que gran parte de esos progresos se han debido a los avances que se han dado en el escenario internacional, en países que han estado a la vanguardia en todo lo relacionado con la preservación de los recursos naturales (OECD/ECLAC, 2014: 54).

Cuando se tiene una pluralidad de emisores de normatividad, sin un esfuerzo por integrar las propuestas planteadas, se debilita la coherencia de los requisitos normativos y se genera incertidumbre, especialmente para las empresas. En ese 
sentido, se vislumbra la necesidad de reestructurar la normatividad ambiental y fortalecerla (OECD/ECLAC, 2014: 54).

Esa confusión que tienen las empresas frente al aparato normativo, incide en que sea visto como algo innecesario y que reporta muy poco valor agregado a las operaciones de la empresa y a su desempeño en general. Junto con esto, otro problema que existe es la divulgación de las políticas públicas, pues se ha apreciado su desconocimiento por parte de una mayoría considerable del empresariado, con lo que se justifica más su falta de aplicación (Hoof, 2005).

A pesar de todo este panorama, Colombia se ha caracterizado por ser un país interesado en el desarrollo de normativa ambiental, y muestra de ello son algunos instrumentos que se han creado con este propósito como la responsabilidad ambiental, los seguros ambientales, los acuerdos voluntarios y el ecoetiquetado (OECD/ECLAC, 2014:61). Estas propuestas se fundamentaron en la premisa de que toda actividad empresarial debe tener adherida una responsabilidad por los efectos que cause en el medio ambiente, de tal forma que la reparación por los daños cometidos debe ser obligatoria. Si bien se trata de un supuesto muy idóneo para la normatividad ambiental, en su aplicación se observaban problemas relacionados con la falta de institucionalidad de los emisores de la norma, así como el hecho de que el mayor incentivo para adoptar estas metodologías estaba en la presión de los mercados y no en la generación de conciencia colectiva frente a lo ambiental.

Esa falta de observancia de la normativa ambiental resulta evidente en el quehacer cotidiano de las empresas. Varias entidades así lo han confirmado: el 80\% de las plantas de tratamiento de aguas servidas no cumplen con los requisitos legales (Sánchez-Triana y Enríquez, 2005 citados en OECD/ECLAC, 2014: 65); alrededor de tres cuartos de los usuarios extraen agua sin autorización (CGR, 2012 citado en OECD/ECLAC, 2014: 65). Como una solución tentativa a la problemática, las autoridades han considerado la necesidad de capacitar a los empleados en un intento por generar la conciencia que se requiere, en términos del cuidado del medio ambiente.

Para garantizar que la implementación de política pública ambiental sea efectiva, el Gobierno ya está desarrollando mecanismos para un monitoreo exhaustivo de las empresas. Ejemplo de ello son herramientas como VITAL y el Registro Único de Infractores Ambientales (OECD/ECLAC, 2014: 67). Otro ejemplo que evidencia la intención por fortalecer la regulación ambiental, es la sanción de la Ley 1453 de 2011, la cual amplió el número de actividades consideradas como delitos ambientales. A pesar de que las multas son más severas, la aplicación ha sido muy restringida (OECD/ECLAC, 2014: 69).

El PND ha tratado de conciliar la necesidad de promover el desarrollo sostenible con el objetivo básico financiero de las empresas, que se concreta en supervivencia y crecimiento de las organizaciones. Lo que se propone es que ambos conceptos se unifiquen y se vean como interdependientes, contrario a lo que ocurre hoy en día, cuando se ven como propuestas en contradicción. Lo anterior guarda una relación muy estrecha con el tipo de cultura predominante en las PYMEs, en el que no puede existir conciencia por lo medioambiental sin que se aprecie un beneficio en lo económico. 
El PND también señala que el problema de formalización de la política pública ambiental en Colombia, se debe a una falta de coherencia entre los planes del sector económico y los objetivos ambientales (OECD/ECLAC, 2014: 86). Si bien se busca la definición de parámetros que conduzcan a un crecimiento sostenible, no existe la suficiente claridad frente a los mecanismos que pueden usarse y las metodologías de seguimiento y control. En últimas, se tiene una intención que no se puede hacer operativa.

Aunque se han intentado varias alternativas como lo es la cooperación intersectorial (a través del Ministerio de Ambiente, Vivienda y Desarrollo Territorial MADS), no se ha logrado un progreso notable debido a la falta de liderazgo. Otro de los grandes inconvenientes es que hay ciertas propuestas que no son de obligatorio cumplimiento por parte de todos los actores sociales, dejando al libre albedrío la opción de contribuir o no a la causa (OECD/ECLAC, 2014: 86).

También se ha sugerido que se implementen nuevos tributos relacionados con el medio ambiente, ya que los recursos recaudados actualmente por dichos impuestos no son suficientes. Como ocurre con la mayor parte de los impuestos, la evasión es una práctica usual y eso va en detrimento de las posibilidades de mejorar los mecanismos de una gestión ambiental adecuada. Aunque se han sugerido impuestos relacionados con el combustible y otros recursos energéticos, la resistencia que la población presentaría sería muy notable y el Gobierno teme que estas medidas puedan producir efectos contrarios a los deseados (OECD/ECLAC, 2014: 88).

El análisis de estas medidas ha conducido a la conclusión de que, en un grado considerable, el gran problema de la política pública ambiental se concentra en el sistema de incentivos que se ofrecen, los cuales son insuficientes para promover la adopción de comportamientos amigables con el ambiente. Las políticas públicas enfocadas a la preservación del medio ambiente, en Colombia o en otra parte del mundo, deben no sólo ser promotoras de un crecimiento sostenible, sino que deben sentar las bases para que los esfuerzos de mejora se concentren en beneficios claros que si bien guardan relación con objetivos de índole financiero, también representan una mayor estabilidad para las compañías que las implementen.

\section{CONSIDERACIONES METODOLÓGICAS}

Para llevar a cabo esta investigación se hizo una exploración bibliográfica, siguiendo un diseño longitudinal, analizando artículos en revistas indexadas desde el año 2000 en el contexto internacional que dieran evidencia para concluir una respuesta al objetivo planteado. Se inició por explorar las teorías que delimitan el entendimiento de la cultura organizacional acorde a la gestión ambiental, tomando como base los planteamientos de Schein (2004), Quinn \& Kimberly (1984) y Jones et al (2005) \& Zammuto et al. (2000). Posteriormente se describió la cultura organizacional en PYMEs relacionando estudios de caso con exploraciones bibliográficas previas sobre el tema; finalmente se revisaron documentos legales (decretos y el Plan Nacional de Desarrollo del gobierno) acerca de las políticas públicas al respecto del cuidado ambiental en Colombia, para relacionarlos con posturas teóricas sobre el deber ser de la política pública en materia ambiental. 
Los documentos citados no tuvieron una restricción de tipo histórico o geográfico, su búsqueda estuvo discriminada con base en la inclusión del tema de la cultura organizacional y su relación con la gestión ambiental, las PYMEs y las políticas públicas; los archivos fueron ubicados en las bases Academic Search Complete (EBSCO), Jstor, Science Direct, Redalyc, Scielo y páginas web de dependencias gubernamentales en Colombia. Los documentos fueron también filtrados por su redacción en idioma inglés y español.

La principal limitación para la construcción de este artículo fue la escasa bibliografía sobre cultura organizacional, gestión ambiental y políticas públicas, aplicados a las PYMEs, temas que se encuentran más ampliamente desarrollados para las grandes empresas. Por otro lado, no se identificaron documentos que indagaran la efectividad de la política pública actual en Colombia en las PYMEs.

\section{RESULTADOS}

\subsection{RELACIÓN DE LAS POLÍTICAS PÚBLICAS Y EL PROTOCOLO DE KYOTO}

Las intenciones del protocolo de Kyoto de reducir las emisiones de gases de efecto invernadero se pueden ver desde dos posturas: la postura ambientalista, donde los esfuerzos se dirigen a la prevención de los efectos del calentamiento global; o pueden verse desde el cumplimiento de una norma de reducción de gases de efecto invernadero que desemboca en la dinamización del mercado de bonos de carbono.

Desde la postura ambiental, surgieron políticas públicas en Colombia encaminadas a la reducción de emisiones y al aumento de la eficiencia energética en la economía; esto se materializa en proyectos en el marco del Mecanismo de Desarrollo Limpio, los cuales deben reducir o capturar gases de efecto invernadero de manera real, medible y de largo plazo (Sierra y Guerrero, 2011). Estas iniciativas son impulsadas desde la expedición de la Ley 697 de 2001, la cual:

- Declara el Uso Racional y Eficiente de la Energía (URE) como primordial para la sostenibilidad ambiental y económica de la nación, creando las condiciones para lograr el desarrollo de proyectos concretos

- Crea del programa de Uso Racional y Eficiente de la Energía y demás formas de energía no convencionales (PROURE) como mecanismo de vigilancia del cumplimiento de la eficiencia en las cadenas energéticas, así como promueve el uso de estas fuentes no convencionales de energía

- Obliga a las empresas de servicios públicos a diseñar programas de URE para los usuarios

- Incentiva la formación e innovación para la consecución del URE

Desde el punto de vista de la regulación, la creación de programas que obligan la reducción de emisiones e impulsan los mecanismos de transformación de aire contaminado en oxígeno, generan en las empresas respuestas que no coinciden con el cuidado ambiental, sino con el cumplimiento de la norma al menor costo posible. Es así como en las economías industrializadas, responsables de la mayor parte de la cuota de emisión de gases de efecto invernadero, se promueve la com- 
pra de bonos de carbono, dinamizando este mercado y haciendo de la iniciativa ambiental un negocio de permisos para la contaminación.

Sin embargo, la dinámica que surgió como consecuencia de la implementación del protocolo de Kyoto coincide con los incentivos financieros necesarios para que las PYMEs implementen políticas de gestión ambiental, ya que a través de la Ley 788 de 2002 se crearon estímulos tributarios para las empresas que implementaran tecnologías limpias o que redujeran sus emisiones de CO2 (comprobado por certificados de reducción de emisiones). Por otro lado, la inversión en mejores prácticas que conlleven a la reducción de emisiones se compensaría con la emisión de bonos de carbono que generarían mayores ingresos para las empresas.

Sin perjuicio de lo anterior, queda la pregunta de si las PYMEs tienen un acceso comprobable a los beneficios generados por la reducción de emisiones, ya que dichos beneficios se obtienen luego de una alta inversión en tecnología y de reducciones de emisiones de gran volumen en cuanto los bonos de carbono se emiten en una escala de toneladas.

\subsection{IMPORTANCIA DE LA CO EN LA POLÍTICA PÚBLICA}

Entendiendo la definición de cultura organizacional y tomando los hallazgos de los autores en este documento acorde a la cultura imperante en las PYMEs, es importante reconocer lo que incentiva a este tipo de empresas a tomar decisiones respecto a la gestión ambiental y las características de los socios gestores y administradores que definen la cultura formal (misión, visión, filosofía y estrategia del ente) e informal (el pensamiento tanto de los líderes como de los empleados).

Según previas investigaciones de los autores, en las PYMEs existe una fuerte tendencia hacia la toma de decisiones (respecto a la gestión ambiental) que benefician financieramente a la compañía, que reducen los costos y en general que incrementan las utilidades, características que fueron ubicadas -en párrafos anteriores- en el cuadrante de procesos internos de Zammuto et al. (2000) y Jones et al. (2005). (Bohórquez y Cendales (2014).

La propuesta de los autores es entender la cultura organizacional de las PYMEs para redirigir la política pública al respecto de la gestión ambiental y lograr que estas empresas integren lineamientos de sostenibilidad a su estrategia de manera constante y eficiente; todo esto en pro del cuidado del ecosistema, asunto contemplado en el Plan Nacional de Desarrollo para Colombia (PND).

El PND (con base en el documento para 2010-2014) no contempla un direccionamiento claro para las PYMEs y tampoco hay evidencia clara hacia un enfoque financiero, lo que demuestra que el proyecto de política actual desconoce las problemáticas propias de este tipo de empresas y por lo tanto la política pública emitida puede no ser efectiva en la implantación de lineamientos ambientales.

La política se dirige entonces hacia la masa completa de empresas que conforman los sectores productivos, indicando lineamientos de manera indistinta para grandes y pequeñas empresas. Del Brío y Junquera (2002) exponen que debe existir una regulación medioambiental específica para las PYMEs en cuanto las políticas públicas benefician a la grande empresa al no hacer esta distinción; Aragón (1998) 
y Aurora y Cason (1995), manifiestan que la gran empresa tiene más ventajas al momento de acceder a programas de administraciones públicas y efectivamente participan más en estos programas (Del Brío y Junquera, 2002, p. 10).

La política pública ambiental, por tanto, debe vincular la diferenciación explícita de política hacia las PYMEs e incentivos financieros que correspondan a la cultura organizacional de este tipo de empresas, características que no se encuentran en los programas de fomento ambiental en Colombia analizados hasta el momento por los autores.

\subsection{REDIRECCIONAMIENTO DE LA POLÍTICA PÚBLICA}

La bibliografía explorada, a pesar de contemplar a las PYMEs como objetivo para el cuidado ambiental, no contempla aspectos de cultura organizacional, por ende, los autores proponen una serie de lineamientos con base en ésta para redireccionar la política pública y lograr mayor efectividad en los objetivos planteados por el PND en la sección ambiental.

En concordancia con lo planteado por Hoof (2005), se aprecia que las PYMEs tienen necesidades de tipo ambiental que se derivan de su quehacer, pero no son plenamente atendidas por problemas relacionados con los altos niveles de informalidad, la poca exigencia de sus mercados objetivos y la baja capacidad de las autoridades ambientales competentes para ejercer control y seguimiento (Hoof, 2005:8). Esta situación ya había sido advertida en el PND al hablar de la carencia de incentivos idóneos para propiciar la adopción de lineamientos ambientales.

Una forma para crear tales incentivos es garantizar mayores fuentes de financiación, programas de acompañamiento en consultoría ambiental y flujos de información apropiados (Hoof, 2005:8). Sin este conjunto de incentivos, difícilmente las PYMEs encontrarán interés en desplegar sus planes estratégicos alrededor de los temas medioambientales.

Sin embargo, al contemplar el tipo de cultura que predomina en estas empresas, en la cual es imprescindible el logro de una mejor posición competitiva, resulta esencial que se evalúen los impactos de la adopción de lineamientos ambientales en términos de un análisis costo-beneficio, con el cual los socios gestores puedan encontrar un sustento adecuado para la toma de decisiones.

Junto con la oferta de estos incentivos, también es importante un fortalecimiento institucional de las entidades encargadas de emitir normatividad ambiental, así como de las entidades encargadas de ejercer vigilancia y control, en el entendido de que la falta de integralidad de las políticas públicas atenta contra los objetivos nacionales de desarrollo sostenible, que han sido manifestados en el PND, a través de las locomotoras de crecimiento.

El camino hacia el desarrollo sostenible, con la adopción de lineamientos ambientales que le es inherente, sólo será posible con el trabajo conjunto de todos los actores sociales involucrados. Para el caso particular de las empresas, la integración debe extenderse a su cultura y sus procesos de planificación estratégica. 
Algunas propuestas en específico son:

- Acorde al cuadrante de procesos internos de Jones et al. (2005) \& Zammuto et al. (2000) (Ver Gráfico 1), las PYMEs que apliquen lineamientos de gestión ambiental deberían tener preferencia en la entrada a cadenas de valor, con el ánimo de incentivar las ventas y lograr permanecer en el mercado a lo largo del tiempo.

- Dado el interés financiero y las dificultades económicas propias de las PYMEs, debe existir programas de crédito exclusivo para PYMEs con bajas tasas de interés para implementación de tecnologías limpias y adecuación de la infraestructura para reducir el impacto ambiental. Estos programas pueden estar condicionados por la eficacia de las empresas en el corto (2 años) y mediano plazo (hasta 5 años).

- Dado que la cultura organizacional en las PYMEs no está dirigida hacia la capacitación voluntaria (propio del cuadrante de relaciones abiertas), debe haber una propuesta desde la regulación que requiera que los socios gestores se capaciten sobre gestión ambiental hacia dentro (como reciclaje o cuidado del agua en las instalaciones), como hacia afuera de la organización (impacto sobre los stakeholders). De manera similar a como las Cámaras de Comercio en Colombia hacen con las medidas sanitarias y de riesgos.

- Sin desconocer los programas de innovación tecnológica del SENA de apoyo y fomento en las PYMEs, estos programas podrían tener extensiones exclusivas para la gestión ambiental que estén subsidiados por el gobierno, sobre todo para sectores de alto impacto ambiental como la agricultura y la marroquinería. Aunque, dado que la cultura de la innovación no es evidente en las PYMEs, estos programas deben incluir incentivos de entrada preferencial a cadenas de valor bajo el uso de certificaciones del Estado.

- Dadas las posibles incoherencias entre la cultura formal e informal en las PYMEs y la información oculta que puede darse al perseguir los incentivos ambientales derivados de los programas de gobierno, es indispensable hacer auditorías ambientales en las PYMEs con el fin de controlar la efectividad de la política pública en la consecución de los objetivos de sostenibilidad ambiental.

Estas propuestas parten de la premisa de que las PYMEs tienen una cultura organizacional en común, pero no desconocen que existen PYMEs que voluntariamente transitan hacia enfoques culturales más amigables con el ambiente. A pesar de la existencia de diversos tipos de empresas, estas propuestas contribuyen de manera transversal a la consecución de los objetivos ambientales propuestos en el PND para Colombia 2010-2014.

Por otro lado, lo expuesto en este documento surge de la brecha entre objetivos ambientales y las estrategias para alcanzarlos, existiendo aun vacíos en la política pública que impiden lograr las metas en los planes gubernamentales. 


\section{CONCLUSIÓN}

Tanto las propuestas contenidas en este documento, como las futuras propuestas de política pública, deben reconocer que las empresas son guiadas por una cultura organizacional que impera en la toma de decisiones empresariales; por lo que el alcance de las políticas debe actuar desde la raíz de las decisiones, no desde las consecuencias de las mismas.

La manera de actuar desde la raíz de las decisiones es comprender la cultura de los socios gestores de las PYMEs y lo que los incentiva, saber qué intereses tienen para promover los aspectos ambientales en sus empresas. También debe hacerse la distinción entre grandes, medianas y pequeñas empresas, en tanto éstas no enfrentan iguales necesidades y no comparten una cultura organizacional en común.

$\mathrm{Al}$ respecto de la cultura organizacional en las PYMEs, los autores hallaron en previos documentos que la cultura predominante en estas empresas corresponde a la cultura descrita en el modelo de procesos internos en la teoría de Jones et al. (2005) \& Zammuto et al. (2000), en el que se da prioridad a la estabilidad y al control permanente. Por ello, cualquier iniciativa de gestión ambiental debe atender los objetivos de crecimiento económico. Si no se diseñan los incentivos adecuados, tales iniciativas no serán consideradas como parte integral de la planeación estratégica.

Por lo tanto, las propuestas al respecto de la política pública deben centrarse en incentivar a los socios desde los beneficios económicos, la reducción de gastos, la inclusión en cadenas de valor y debe haber una presión adicional desde la regulación dirigida hacia la capacitación en tanto según la cultura organizacional en las PYMEs no existe una capacitación voluntaria de parte de los socios hacia los empleados y para ellos mismos.

Además, si bien existen políticas públicas con incentivos de dicha naturaleza, estos ignoran la accesibilidad de las empresas a hacer uso de los incentivos, en cuanto exigen inversiones altas en tecnología y resultados a gran escala, los que pueden ser inalcanzables para las PYMEs.

Finalmente, para velar por el alcance de los objetivos gubernamentales en materia ambiental debe haber auditoría constante de parte de entes públicos para comprobar que las PYMEs utilizan de manera adecuada los incentivos de la administración pública.

A partir de los hallazgos y propuestas de este documento, futuras investigaciones podrían proponer estudios de caso sobre la efectividad de las políticas públicas ambientales en PYMEs colombianas; además se podría verificar la implicación de la cultura organizacional de estas empresas en el logro de la sostenibilidad ambiental. 


\section{REFERENCIAS}

1. ACQUATELA, J. (2001). Fundamentos económicos de los mecanismos de flexibilidad para la reducción internacional de emisiones en el marco de la Convención de Cambio Climático (UNFCCC). Medio Ambiente y Desarrollo, 1-41.

2. AKTOUF, O. (2002). El simbolismo y la "cultura organizacional", de los abusos conceptuales a las lecciones de campo. Revista de la Escuela de Administración. Universidad EAFIT, (1), Medellín, 63-93.

3. BALZAROVA, M.A.; CASTKA, P.; BAMBER, C.; SHARP, J. (2006), How organisational culture impacts on the implementation of ISO 14001:1996-a UK multiple-case view, Journal of Manufacturing Technology Management; Vol. 17 No. 6, pp. 89-103.

4. BIANCHI, R. Y NOCI, G. (1998). “GREENING SMEs Competitiveness. Small Business Economics, 11 (3), 269-281.

5. CAMERON, K. \& QUINN, R. (1999). Diagnosing and changing organizational culture. Base on the Competing Values Framework. Revised Edition. The Jossey-Bass. Business \& Management Series. United States of America. $242 \mathrm{p}$.

6. COLOMBIA (1997), Constitución Política, Bogotá, Legis.

7. DEL BRÍO, J. Y JUNQUERA, B. (2002). Gestión medioambiental en la PYME: consideraciones para las políticas públicas. ICE Tribunal de Economía, v (798), 191-206.

8. FRASSA, J. (2011). Cultura organizacional: conceptualizaciones y metodologías detrás de un concepto complejo. En: Dirección y Organización. Revista de Ingeniería de Organización, (44), 74-85

9. GÁLVEZ, E. Y GARCÍA, D. (2011). Cultura organizacional y rendimiento de las MIPYMEs de mediana y alta tecnología: un estudio empírico en Cali, Colombia. Cuadernos de Administración, 24 (42), 125-145.

10. GARCÍA, L., \& CUESTA, C. (2007). El Protocolo de Kyoto y los costos ambientales. Revista del Instituto International de Costos, 9-31.

11. HERRERA, J., LARRÁN, M. Y MARTÍNEZ-MARTÍNEZ, D. (2012). Relación entre responsabilidad social y performance en las pequeñas y medianas empresas: Revisión bibliográfica. Cuadernos de Gestión, 13(2), 39-65.

12. HOFSTEDE, G., NEUIJEN, B., DAVAL, D. Y SANDERS, G. (1990). Measuring organizational culture: A qualitative and quantitative study across twenty cases. En: Administrative Science Quarterly, vol. 35, 286-316

13. HOOF, B. (2005). Políticas e instrumentos para mejorar la gestión ambiental de las PYMEs en Colombia y promover su oferta en materia de bienes y servicios ambientales. CEPAL-SERIE Medio Ambiente y Desarrollo, 94, 3-77.

14. HOWARD-GRENVILLE, J. (2006). Inside the black box: How organizational culture and subcultures inform interpretations

15. JENKINS, H. (2009). A 'business opportunity' model of corporate social responsibility for small- and medium-sized enterprises. Business Ethics: A European Review, 18 (1), 21-36. 
16. LAFERRIERE, R. (2008). El mecanismo de desarrollo limpio del Protocolo de Kyoto. Buenos Aires: Ediciones Lulu.

17. LAMARCA JUNIOR, M. R., \& LEITE DA SILVA, C. R. (2009). O mercado do carbono como instrumento de conservação da floresta Amazônica. Revista de Economia Mackenzie, 63-83.

18. LEY 697 DE 2001. Presidencia de la República. Colombia.

19. LEY 788 de 2002. Presidencia de la República. Colombia.

20. LINNENLUECKE, M. \& GRIFFITHS, A. (2010). Corporate sustainability and organizational culture. Journal of World Business, 45, 357-366.

21. MONROY, N., \& AGUIRRE, A. (2003). El Protocolo de Kyoto: ¿una oportunidad para la industria Colombiana? Revista de Ingeniería, 108-116.

22. OECD/ECLAC - Organización para la Cooperación y el Desarrollo Económico; Comisión Económica para América Latina y el Caribe. (2012). Perspectivas económicas de América Latina 2013: Políticas de PYMEs para el cambio estructural. París, Francia: OCDE

23. OECD/ECLAC - Organización para la Cooperación y el Desarrollo Económico; Comisión Económica para América Latina y el Caribe. (2014). Latin American economic Outlook 2014: Logistics and competitiveness for development. París, Francia: OCDE

24. PLAN NACIONAL DE DESARROLLO 2010-2014. Presidencia de la República. Colombia.

25. RODRÍGUEZ, R. (2009). La cultura organizacional: un potencial activo estratégico desde la perspectiva de la administración. Invenio, 12 (22), 67-92.

26. SAGRERA, L. V. (2011). Los avances y progresos de la República Argentina en la disminución de las emisiones de $\mathrm{CO}_{2}$, luego de la vigencia del protocolo de Kyoto. Provincia, 73-91.

27. SCHEIN, E. (2004). Organizational Culture and Leadership. Third Edition. Jossey Bass. United States of America. $437 \mathrm{p}$.

28. SCHLIERER, WERNER, SIGNORI, GARRIGA, WELTZIEN, VAN ROSSEM \& FASSIN. (2012). ¿How do European SME owner-managers make sense of "Stakeholder Management"? Insights from a cross-national study. Journal of Business Ethics, 109, 39-51.

29. SIERRA, F. Y GUERRERO, A. (2011). Bonos de carbono y programa de eficiencia energética en Colombia. Revista Voces en el Fénix. Universidad de Buenos Aires, (10), Buenos Aires, 92-99

30. SMIRCICH, L. (1983). Concepts of Culture and Organizational Analysis. En: Administrative Science Quarterly, vol. 28(3), 339-358

31. UHLANER, L., BERENT-BRAUN, M., JEURISSEN, R. \& WIT, G. (2011). Beyond size: predicting engagement in environmental management practices of Dutch SMEs. Journal of Business Ethics, 109 (4), 411-429. 